\title{
Methicillin-resistant Staphylococcus aureus in poultry- an emerging concern related to future epidemic.
}

Zaytoon Zaheer ${ }^{1}$, Sajjad Ur Rahman' ${ }^{1}$, Iqra Zaheer ${ }^{2}$, Ghazanfar Abbas ${ }^{1}$, Tayyaba Younas

${ }^{1}$ Institute of Microbiology, Faculty of Veterinary Science, University of Agriculture Faisalabad;

${ }^{2}$ Department of Pathology, Faculty of Veterinary Science, University of Agriculture Faisalabad.

*Corresponding author: zaytoonzaheer@gmail.com

Abbreviated title: MRSA of Poultry

This is an open access article distributed under the Creative Commons Attribution License, which permits unrestricted use, distribution, and reproduction in any medium, provided the original work is properly cited.

\section{ARTICLE DETAILS}

Article history:

Received 22 January 2017

Accepted 03 February 2017

Available online 05 February 2017

Key words:

MRSA; poultry; zoonosis; colonization

\section{ABSTRACT}

Methicillin resistant - Staphylococcus aureus (MRSA) is an organism responsible for causing deadly infections in man, poultry and several other animal species. Ever since the discovery of MRSA in decade of 1960's, it has been isolated and reported in live poultry birds and their meat products worldwide. Strikingly, MRSA recovered from poultry share a common ancestry with MRSA isolates of human and animal origin. Furthermore, MRSA colonizes the skin and mucous membranes of both man and poultry. This colonizing ability of MRSA is not only a cause of nonresponsive infections in poultry but it can also be transferred to the humans handling birds at farms and slaughter houses. Moreover, the consumers of poultry meat can also acquire its infection. The cases of cross infections of MRSA between poultry and human has been reported. However, the most important difficulty to control MRSA and its interspecies transmission is the irrational use of antimicrobial drugs and inadequate biosecurity measures. Therefore, rational usage of antimicrobials and good biosecurity measures need to be adopted for prevention and control of zoonotic MRSA infections. This paper reviews the existing status of MRSA in poultry as a possible threat of zoonosis

\section{Introduction}

Staphylococcus aureus is a non-motile, non-spore producer, gram positive coccus. It was discovered when Alexander Ogston in 1880 made an attempt to demonstrate the role of bacteria in infecting wounds and causing septicemia. During his research he had observed 88 human pus samples under the microscope and found the presence of Gram-positive cocci in these samples (later on named as Staphylococci, a member of firmicutes). A species of genus Staphylococcus i.e. Staphylococcus aureus is one of the normal inhabitants of skin and nares (man and animals) which may also act as pathogen under certain circumstances. (Herold et al, 1998; Vengust et al, 2006, Licitra, 2013).

For many years, treatment of wounds infected with Staphylococci involved the application of phenol. This treatment approach was first used by Lister and later on Ogston routinely used the same method (Herold et al, 1998).

\section{History of MRSA emergence:}

Penicillin was discovered in 1940s and was hailed as the miracle drug but in 1961 (only 2 years following the discovery of methicillin: a penicillin resistant to penicillinase), $S$. aureus became resistant to methicillin by acquiring mec A gene and by 1950 the frequency of resistance was significant hence it arose the need of implementing an alternative treatment strategy (Lowy, 2003).

In the mean while some strains of S. aureus produced a modified penicillinbinding protein (Penicillin binding protein $2 \mathrm{a}$ ) and became resistant to semi-synthetic $\beta$-lactam antimicrobials (BLAs) which were at that time being used for treatment of $S$. aureus infections after observing resistance to penicillin and those resistant strains were termed as methicillin-resistant Staphylococcus aureus (MRSA) first observed in early decade of 1960's (Giannoudis et al, 2005). At present in addition to BLAs, MRSA may also show resistance to common antimicrobials, e.g. macrolides, lincosamides, streptogramin, tetracycline, gentamicin (Delorme et al, 2009).

After the discovery of MRSA's occurrence as a nosocomial pathogen, several hospital acquired MRSA (HA-MRSA) clones infected man worldwide. However, since the 1990s, community-acquired MRSA (CA-MRSA) clones were also found to be spreading globally. In this regard MRSA spread is now observed to occur beyond hospital facilities, becoming a significant public health and zoonotic issue due to which it is now also regarded as a community associated pathogen (Vengust et al, 2006; Persoons et al, 2009; Lee, 2003; Leonard, 2006).

Besides human MRSA has also been isolated from several animal species and such isolates are a source of resistant zoonosis particularly this organism is prevalent in food animals because of the irrational use (usage below therapeutic level) of antimicrobials for preventing diseases and growth promotion (Gilchrist et al, 2007).

Like other food animals MRSA can colonize the birds treated with antibiotics however a portion of MRSA in poultry can be due to human (e.g. due to MRSA infected farm and slaughter house workers) contamination of birds and their products (Waters, 2011).

\section{Importance of Penicillin Binding Protein 2a (PBP 2a) for MRSA:}

Methicillin resistant Staphylococcus aureus is among the most important pathogens in human and veterinary health care facilities due to which it is globally considered as a serious health concern (Goetghebeur et al, 2007). It has the ability to produce a different penicillin-binding protein (PBP) i.e PBP 2a which shows comparatively low susceptibility to most of the beta-lactam antibiotics. The mecA gene is (responsible for encoding PBP2 a) $2.1 \mathrm{~kb}$ long and is present on a mobile Staphylococcal genomic island i.e staphylococcal cassette chromosome mec (SCCmec). There are seven main variants of SCCmec (type I to VII). The synthesis of PBP, called PBP-2a (Hartman and Tomasz, 1986), PBP2' (Murakami, 1987) or MRSA-PBP (Song, 1987), is considered to be the most important if not the only mean of conferring high resistance to $\beta$-lactams. Moreover, absence of resistance to methicillin in S. aureus is frequently seen associated to the absence of PBP (Ubukata, 1985).

\section{Host Range:}

Staphylococcus aureus can be found to colonize skin and mucosal membranes of human as well as animals (e.g. cat, dog, horse, pig, farm animals, psittacine birds and poultry) as normal flora but may also cause resistant and lethal infections in the host (Leonard, 2006; Walther et al, 2008; Persoons et al, 2009).

\section{Significance of MRSA in Poultry:}

S. aureus is not only a normal inhabitant of the mucosa of all mammalian species but is also found to colonize the mucosal membranes in domestic poultry (Hajek et al, 1988) and commercially raised populations of poultry birds (Witte et al, 1977).

The first report on occurrence of MRSA in poultry was from South Korea, where it was isolated from arthritis affected birds. Molecular typing of the strains revealed a common ancestry of the poultry strains with those isolated from humans and bovine milk samples (Lee, 2003). Later on several times MRSA has been isolated from live poultry birds as well as from poultry meat (Kitai et al, 2005). Staphylococcal species in poultry are generally regarded as harmless normal inhabitants but these species can be pathogenic under certain conditions causing opportunistic infections. MRSA from poultry birds may not only infect the bird itself but also poses a zoonotic risk for humans handling poultry (Persoons et al, 2009; Lee, 2003; Oke and Oke, 2013). 
In a study conducted at a poultry farm located in South West Nigeria, it was found that out of 100 cloacal swabs collected from 6-week old broiler birds (showing the symptoms of diarrhea) over $90 \%$ of the cloacal swabs yielded MRSA. Furthermore, nasal swabs from 25 attendants (with symptoms of cough and catarrh) and 5 attendants (without symptoms of cough and catarrh) working at the poultry farm were collected and MRSA was isolated from all the attendants showing the symptoms of cough and catarrh. Incidence of MRSA was $83.3 \%$ in the poultry attendants, and $95 \%$ in the poultry was recorded. Infection of MRSA in poultry may be acquired from man and may also spread from poultry birds to the workers at farm. MRSA from poultry can be transferred to man not only by digestion of contaminated meat but also through contact with live birds and their fecal material (Oke and Oke, 2013). MRSA excreted by birds in feces can also exist in poultry litters (Graham et al, 2009)

In another research study methicillin-resistant Staphylococcus aureus (MRSA) isolation from several food products was reported. It was found that slaughtering of MRSA-infected animals may cause the contamination of carcasses. To estimate the occurrence of MRSA in 2217 raw meat samples of beef, veal, pork, chicken, lamb/mutton, turkey, and fowl were collected. MRSA was isolated from 264 meat samples and isolation percentage was: beef $(10.6 \%)$, chicken $(16.0 \%)$, veal $(15.2 \%)$, lamb and mutton $(6.2 \%)$ turkey $(35.3 \%)$, pork $(10.7 \%)$, fowl $(3.4 \%)$ and game $(2.2 \%)$. It was concluded that further research work is required to identify the routes of transmission in relation to meat and meat products to limit the spread of MRSA (Boer et al, 2009)

In Belgium cloacal and nasal samples of seventy-five broilers and fifty laying hens were collected from randomly selected farms and fifteen isolates of MRSA were obtained from broilers and no isolate was obtained from laying hen samples probably because of a limited usage of antimicrobials in layers (Persoons et al, 2009)

A research study was conducted to determine the prevalence and antibiotic resistance patterns of Staphylococcus aureus isolated from meat and chicken samples. For this purpose, one hundred and fifty samples of calf/ lamb meat (mince and chunks) and chicken parts (giblets, carcass) were collected and eighty (including 26 isolates of poultry origin) S. aureus strains were identified. The overall methicillin resistance rate for eighty isolates of S. aureus during the research was found to be $67.5 \%$. However chicken carcass and chicken giblets showed a methicillin resistance of $76.4 \%$ and $55.5 \%$ respectively (Gundogan et al, 2005).

In Qena governorate 125 poultry samples were collected during 2012 for analyzing the presence of methicillin-resistant Staphylococcus aureus (MRSA). During the study samples were collected from freshly slaughtered whole chicken carcasses (25/125), chicken luncheon (25/125), chicken portions (25/125), chicken sausages $(25 / 125)$ and chicken burgers (25/125). Isolation percentage was found to be $44 \%(11 / 25), 52 \%(13 / 25)$, $40 \%(10 / 25), 24 \%(6 / 25)$ and $44 \%(11 / 25)$ for freshly slaughtered whole chicken carcasses, chicken portions, chicken luncheon, chicken sausages and chicken burgers respectively. At the end of the study it was found that there are higher contamination rates associated with raw poultry meat and relatively lower rate of contamination were reported in poultry meat products treated with heat and preservatives. However, contaminated poultry meat and its products are now regarded as one of the important sources of MRSA transmission to humans due to unhygienic poultry slaughter houses and food preparing establishments (Karmi, 2013).

The first report of presence of MRSA in commercial raw poultry meat in Japan was recorded in 2004. Four hundred and forty-four raw poultry meat samples were collected and two mec A positive MRSA strains were isolated (Kitai et al, 2004).

To detect the zoonotic potential of MRSA particularly isolated from food animals a study was conducted in Korea from May 2001 to April 2003 during which one thousand nine hundred and thirteen samples of feces, feed material, milk, joint, uterus, trachea, and meat were collected from cattle, poultry, and pigs. 421 isolates of $\mathrm{S}$. aureus were obtained out of which 28 isolates of S. aureus were resistant to a concentration more than $2 \mu \mathrm{g} /$ $\mathrm{ml}$ of oxacillin. Fifteen (12 from dairy cows and 3 from poultry) of these 28 isolates were mec A positive (Lee, 2003).

To identify the presence of MRSA in turkey, broiler barns as well as in soil and air of the farm's vicinity, a study was conducted in Germany. In total two broiler and five turkey farms were sampled three and four times, respectively. During one fattening period a longitudinal study was designed and samples were obtained from choana and skin of broilers and turkey, air, boot swabs of ground surfaces at different distances from the barn. As a result of longitudinal study MRSA was found in the air outside two turkey barns and also over the ground surface on downwind side of many (44.4\%) broiler and turkey farms. Furthermore, a cross-sectional study was conducted on the farms (including four broiler and five turkey farms) during the last third of the fatting period.

During the cross-sectional study MRSA was detected in the air samples of barns (7 of $9,77.8 \%$ ), as well as samples collected from animals, with detections levels of 62 to $77 \%$ in turkey and 50 to $54 \%$ in broiler farms (Friese et al, 2013).

A research study was planned in US to detect multidrug resistant $\mathrm{S}$. aureus including MRSA in meat and poultry samples. Out of 136 samples S. aureus contaminated $47 \%$ of the samples and multidrug resistance was detected in $52 \%$ of the isolates. Precisely MRSA was detected in one sample from each of turkey, beef and pork (Waters et al, 2011).

\section{Epidemiology:}

Epidemiology of MRSA is changing world wide (Herold, 1998; Boyce, 1998) due to the spread of MRSA beyond hospital premises. Recently in some parts of the world MRSA is recognized as a community pathogen (Mitchell et al, 1996).

MRSA prevalence in human population can be around 60\% (Hafiz et al, 2002) while true prevalence studies regarding MRSA in poultry are scarce. However, new reservoirs for MRSA may include poultry meat (Rizek et al, 2011; Lee, 2003; Lee, 2006; Kitai et al, 2005) as well as live poultry birds (Waters et al, 2011).

\section{Transmission \& Zoonosis:}

Close contact of human beings with animals may provide an opportunity for transmission of the infection between species. Once the infection is acquired, horizontal transmission may occur between animals or man and also their families can acquire the organism. However, MRSA can be transmitted as follows (Coia et al, 2006; Oke and Oke, 2013):

- Patients infected with MRSA during their stay at the health care facilities after discharge from the hospitals can be a source of spread for healthy population;

- Health workers who have acquired the infection from the infected patients may also transmit the infection to the non-infected individuals of the population;

- In a population the presence of individuals infected with MRSA may be considered as a source of infection for the non-infected members of the community;

- MRSA acquired by anthroposis (transfer from human to animals) or zoonosis (transfer of infection from animals to human due to handling of animals e.g. live birds, poultry litter and meat) is a cause of interspecies spread of the infection.

\section{Disease:}

In healthy individuals (man and animals) a breach in skin /mucous membranes or acquisition of the organism from infected individuals may provokes Staphylococcus aureus particularly MRSA to cause life-threatening staphylococcal ailments. As in case of human beings, poor condition of animal's skin also favors staphylococcal colonization. Mostly these ailments include food poisoning, pneumonia, septicemia and other infections of invasive nature (Boucher and Corey, 2008).

Particularly in birds it may cause suppurative disease, omphalitis, urinary tract infection, otitis, pyoderma and arthritis which are linked to several bacterial virulence factors e.g. enzymes and toxins (Bergmann et al, 1980). However, in man MRSA is frequently found in patients of staphylococcal food poisoning, post-operative wound infections, and pneumonia (Horan et al, 1988).

\section{Prevention and control:}

Mass awareness campaigns, general public education, common sense and good hygiene are keys to prevent MRSA infections, especially in veterinary medicine. The prevention and control of MRSA transmission may include (Morgan, 2008; Coia et al, 2006):

- Consider MRSA infection as a possibility in individuals experiencing post-surgical infections particularly those which do not respond to antimicrobial treatment;

- Individuals with suspected MRSA infection should be segregated and samples for bacteriological examination and susceptibility testing for antimicrobials should be procured from all such cases before instituting antimicrobial therapy; 
- Confirmed cases of MRSA should be handled by wearing gloves and equipment used in such cases which must be disposed or autoclaved afterwards;

- Health care personnel should wash hands properly with alcoho based sanitizers after handling suspected or confirmed cases.

- Co-operation between veterinary and medical professionals is required to check zoonosis.

- Veterinarian should inform owners regarding the risk of acquiring MRSA infection due to contact with already infected animals.

\section{Conclusion:}

MRSA is not only a nosocomial but also a community acquired and zoonotic infection. Shedding of the organism from asymptomatic human, animals, birds, cats, dogs etc. carriers along with selection pressures due to injudicious usage of antimicrobial feed additives and antibiotics may be among the factors which contribute to the establishment of MRSA in the food chain. Furthermore, new strains of MRSA are appearing to evolve in the animals with time and are a source of occupational exposure for humans Workers at farm handling the birds and farm litter can transmit to or acquire MRSA from birds. The role of slaughter house worker in contaminating the meat or handling contaminated meat and products cannot be neglected in possible spread of MRSA. The birds should be handled by wearing gloves and masks to avoid MRSA transmission in either case. The infected poultry flock and its litter must be properly disposed.

The true magnitude of the problem is still not well documented. Hence, there is need of well documented data to have thorough knowledge of true prevalence rates in poultry flock and its ability to evolve with time. Failures to get rid of MRSA carriage from man may possibly be due to reacquisition of the organism from other human beings or animals in close contacts. Hence, the possibility of an animal reservoir must be considered in cases of relapse of the infection, and in proven cases decolonization of all reservoirs must be exercised. A history of close contact to animals and birds may be sought in the cases of repeated and inexplicable failures of MRSA decolonization man and in situations where sharing of MRSA between human and animal is suspected, a combined approach regarding diagnosis and treatment must be developed.

However, there is absolutely no substitute for rational usage of antimicrobials and good biosecurity measures, at the household plus daily farm operations conducted at poultry farm, slaughterhouses, meat processing operations and also at human as well as in veterinary health care facilities.

\section{References:}

Bergmann, V., Kohler, B. and Vogel, K. (1980) Staphylococcus aureus infection in chickens in industrialized poultry units. Archiv fur Experimentelle Veterinarmedizin 34: 891-903.

Boer, Ede., Zwartkruis-Nahuis, J.T.M., Wit, B., Huijsdens, X.W., Neeling, A.J., Bosch, T., van Oosterom, R.A.A., Vila, A. and Heuvelink, A.E. (2009) Prevalence of methicillin-resistant Staphylococcus aureus in meat. International Journal of Food Microbiology 134: 52-56.

Boucher, H.W. and Corey, G.R. (2008) Epidemiology of methicillin-resistant Staphylococcus aureus. Clinical Infectious Diseases 46: 344-349.

Boyce, J.M. (1998) Are the epidemiology and microbiology of methicillin resistant Staphylococcus aureus changing?. Journal of the American Medical Association 279: 623-624.

Coia, J.E., Duckworth, G.J., Edwards, D.I., Farrington, M., Fry, C., Humphreys, H., Mallaghan, C. and Tucker, D.R. (2006) Guidelines for the control and prevention of meticillin-resistant Staphylococcus aureus (MRSA) in healthcare facilities. Journal of Hospital Infection 63: 1-44.

Delorme, T., Rose, S., Senita, J., Callahan, C. and Nasr, P. (2009) Epidemiology and susceptibilities of methicillin-resistant Staphylococcus aureus in Northeastern Ohio. The American Journal of Clinical Pathology 132: 616687.

Friese, A., Schulz, J., Zimmermann, K., Tenhagen, B.A., Fetsch, A., Hartung, J. and Röslera, U. (2013) Occurrence of Livestock-Associated MethicillinResistant Staphylococcus aureus in Turkey and Broiler Barns and Contamination of Air and Soil Surfaces in Their Vicinity. Applied and Environmental Microbiology 79: 2759-2766.

Giannoudis, P.V., Parker, J. and Wilcox, M.H. (2005) Methicillin resistant Staphylococcus aureus in trauma and orthopaedic practice. Journal of bone and joint surgery 87-B: 749-754.

Gilchrist, M.J., Greko, C., Wallinga, D.B., Beran, G.W., Riley D.G. and Thorne, P.S. (2007) The potential role of concentrated animal feeding operations in infectious disease epidemics and antibiotic resistance. Environmental Health Perspectives 115: 313-316.

Goetghebeur, M., Landry, P.A., Han, D. and Vicente, C. (2007) Methicillin- resistant Staphylococcus aureus: A public health issue with economic consequences. The Canadian Journal of Infectious Diseases \& Medical Microbiology 18: 27-34.

Graham, J.P., Evans, S.L., Price, L.B. and Silbsergeld E.K. (2009) Fate of antimicrobial-resistant enterococci and staphylococcus and resistance determinants in stored poultry litter. Environmental Research 109: 682689.

Gundogan, N., Citak, S., Yucel, N. and Devren, A. (2005) A note on the incidence and antibiotic resistance of Staphylococcus aureus isolated from meat and chicken samples. Meat Science 69: 807-810

Hafiz, S., Hafiz, A.N., Ali, L., Chughtai, A.S., Memon, B., Ahmed, A., Hussain, S., Sarwar, G., Mughal, T., Awan, A., Zaki, K. and Fareed, A. (2002) Methicillin resistant Staphylococcus aureus: a multicentre study. Journal of Pakistan Medical Association 52: 31- 32

Hajek, V., Horak, V. and Balusek, J. (1988) Phage typing coagulase-positive staphylococci from rooks and gulis. Research in Veterinary Science 44: 247-250.

Hartman, B. J. and Tomasz, A. (1986) Expression of methicillin resistahce in heterogeneous strains of Staphylococcus aiureuts. Antimicrobial Agents and Chemotherapy 29: 85-92.

Herold, B.C., Immergluck, L.C., Maranan, M.C., Lauderdale, D.S., Gaskin, R.E., Boyle-Vavra, S., Leitch, C.D. and Daum, R.S. (1998) Communityacquired methicillin-resistant Staphylococcus aureus in children with no predisposing risk. Journal of the American Medical Association 279: 593598.

Horan, T., Culver, D., Jarvis, W., Emori,G., Banerjee, S., Martone, W. and Thornsberry, C. (1988) Pathogens causing nosocomial infections: preliminary data from the national nosocomial infections surveillance system. Antimicrobic Newsletter 5: 65-67.

Oke, A. J. and Oke, A.A. (2013) Incidence of Methicillin-Resistant Staphylococcus aureus (MRSA) In a Small Poultry in South West, Nigeria. IOSR Journal of Agriculture and Veterinary Science 5: 2319-2372.

Karmi, M. (2013) Prevalence of methicillin-resistant Staphylococcus aureus in poultry meat in Qena, Egypt. Veterinary World 6: 711.

Kitai, S., Shimizu. A., Kawano, J., Sato, E., Nakano, C., Uji, T. and Kitagawa, H. (2005) Characterization of methicillin-resistant Staphylococcus aureus isolated from retail raw chicken meat in Japan. The Journal of Veterinary Medical Science 67: 107-110.

Lee, J.H. (2003) Methicillin (oxacillin)-resistant Staphylococcus aureus strains isolated from major food animals and their potential transmission to humans. Applied and Environmental Microbiology 69: 6489-6494.

Lee, J.H. (2006) Occurrence of methicillin-resistant Staphylococcus aureus strains from cattle and chicken, and analyses of their mecA, mecR1 and mecI genes. Veterinary Microbiology 114: 155-159.

Leonard, F. (2006) MRSA in domestic animals - an update. Irish Veterinary Journal 59: 90.

Licitra, G. (2013) Etymologia: Staphylococcus. Emerging Infectious Diseases 19:1553.

Lowy, F.D. (2003) Antimicrobial resistance: the example of Staphylococcus aureus. Journal of Clinical Investigation 111: 1265-1273.

Mitchell, J.M., MacCulloch, D. and Morris, A.J. (1996) MRSA in the community. New Zealand Medical Journal 109: 411.

Morgan, M. (2008) Methicillin-resistant Staphylococcus aureus and animals: zoonosis or humanosis? Journal of Antimicrobial Chemotherapy 62: 1181 $-118$.

Murakami, K., Nomura, K., Doi, M. and Yoshida, T. (1987) Production of low-affinity penicillin-binding protein by low- and high-resistance groups of methicillin-resistant Staphylococcus aureus. Antimicrobial Agents and Chemotherapy 31: 1307-1311.

Persoons, D., Van Hoorebeke, S., Hermans, K., Butaye, P., de Kruif, A., Haesebrouck, F. and Dewulf, J. (2009) Methicillin-Resistant Staphylococcus aureus in Poultry. Emerging infectious diseases. 15: 452-453.

Rizek C.F., Matté, M.H., Dropa, M., Mamizuka, E.M., de Almeida, L.M., Lincopan, N., Matté , G.R. and Germano, P.M. (2011) Identification of Staphylococcus aureus carrying the mecA gene in ready-to-eat food products sold in Brazil. Foodborne pathogens and disease 8: 561-563.

Song, M. D., Wachi, M., Doi, M., Ishino, F. and Matsuhashi, M. (1987) Evolution of an

inducible penicillin-target protein in methicillin-resistant Staphylococcus aureus by gene fusion.

Federation of European Biochemical Societies Letters 221: 167-171.

Ubukata, K., Yamashita, N. and Konno, M. (1985) Occurrence of a 1-lactaminducible penicillin-binding protein in methicillin resistant staphylocci. Antimicrobial Agents and Chemotherapy 27: 851- 857.

Vengust, M., Anderson, M.E.C., Rousseau J. and Weese, J.S. (2006) Methicillinresistant staphylococcal colonization in clinically normal dogs and horses in the community. Letters in Applied Microbiology 43: 602-606. 
Walther, B., Wieler, L.H., Friedrich, A.W., Hanssen, A., Kohn, B., Brunnberg, L. and Lübke-Becker, A. (2008) Methicillin-resistant Staphylococcus aureus (MRSA) isolated from small and exotic animals at a university hospital during routine microbiological examinations. Veterinary Microbiology 127:171-178.

Waters, A.E., Contente-Cuomo, T., Buchhagen, J., Liu C.M., Watson, L., Pearce, K., Foster, J.T., Bowers, J., Driebe, E.M., Engelthaler, D.M., Keim, P.S. and Price, L.B. (2011) Multidrug-resistant Staphylococcus aureus in US meat and poultry. Clinical Infectious Diseases 52: 1227-1230.

Witte, W., Hummel, R., Meyer, W., Exner, H. and Wundrak, R. (1977) On the ecology of Staphylococcus aureus: characterization of strains from chicken. Zeitschrift Fur Allgemeine Mikrobiologie 17: 639-644. 